\title{
A large-scale demonstration of SDN-based handover management in IEEE 802.11 networks
}

\author{
Ensar Zeljkovic, Johann M. Marquez-Barja, Steven Latré \\ University of Antwerp - imec, IDLab research group \\ Antwerp, Belgium \\ name.lastname@uantwerpen.be
}

\begin{abstract}
The density of Wi-Fi at professional environments, such as offices, is resulting in increased strain on the management of Wi-Fi networks, of which handovers are an essential part of. The centralized approach of SDN allows taking more intelligent decisions, as opposed to per device optimizations. There is thus an increased interest in SDN-based Wi-Fi management, especially for handovers. Several proposals for SDNbased handover algorithms are being investigated and there is a need for actually validating them. In this demo, we will showcase a Wi-Fi SDN framework, on top of which a number of decision-making handover algorithms can be used. This framework is used in a real-life, large scale Wi-Fi testbed. The framework allows for different decision-making handover algorithms to be created and validated on large scale.
\end{abstract}

\section{CCS CONCEPTS}

- Networks $\rightarrow$ Network resources allocation; Network algorithms;

\section{KEYWORDS}

IEEE 802.11, SDN, framework, handover, algorithm

\begin{abstract}
ACM Reference Format:
Ensar Zeljkovic, Johann M. Marquez-Barja, Steven Latré. 2018. A large-scale demonstration of SDN-based handover management in IEEE 802.11 networks. In SIGCOMM Posters and Demos '18: ACM SIGCOMM 2018 Conference Posters and Demos, August 20-25, 2018, Budapest, Hungary. ACM, New York, NY, USA, 3 pages. https://doi. org/10.1145/3234200.3234223
\end{abstract}

Permission to make digital or hard copies of all or part of this work for personal or classroom use is granted without fee provided that copies are not made or distributed for profit or commercial advantage and that copies bear this notice and the full citation on the first page. Copyrights for components of this work owned by others than ACM must be honored. Abstracting with credit is permitted. To copy otherwise, or republish, to post on servers or to redistribute to lists, requires prior specific permission and/or a fee. Request permissions from permissions@acm.org.

SIGCOMM Posters and Demos '18, August 20-25, 2018, Budapest, Hungary

(c) 2018 Association for Computing Machinery.

ACM ISBN 978-1-4503-5915-3/18/08 .. \$15.00

https://doi.org/10.1145/3234200.3234223

\section{INTRODUCTION}

One of the biggest challenges in IEEE $802.11 \mathrm{Wi}$-Fi networks [2] is handovers. Handing over a mobile node from one access point (AP) to another is a time consuming process resulting in network performance degradation, thus reducing the enduser's quality of experience. In standard Wi-Fi, it can take up to 4 seconds for a mobile node to re-associate to another $\mathrm{AP}$, during which there is no data transfer, [5], immediately impacting the quality of service (QoS) parameters, such as throughput, latency, etc.

More intelligent decisions regarding handovers can be made by using the centralized approach of software defined networks (SDNs), rather then performing per device optimizations. Having the handover decision-making process at a centralized location enables utilizing all the relevant network side information to build decision-making handover algorithms capable of reducing the impact of a handover on the QoS parameters. There is, therefore, a need to validate these algorithms.

In this demo, we will showcase a complete Wi-Fi SDN framework for validating decision-making handover algorithms on a large scale testbed, where up to 60 fixed wireless nodes and up to 17 mobile wireless robots can be used. Both the testbed and the framework are equipped with a visual interface. We aim to i) demonstrate the features and usability of the SDN framework, which offers a rich set of network monitoring information; and to ii) visualize and test the performance of four different handover algorithms running on top of a real-life testbed in complex scenarios including mobility.

\section{SDN AND HANDOVER ALGORITHMS}

As the underlaying wireless SDN platform, we rely on 5GEmPOWER, [3], which leverages the concept of a virtual $\mathrm{AP}$, enabling seamless handovers that show no impact on the throughput of a mobile node during a handover, [4]. We use this platform as a baseline to further build our seamless handover solution on top of it. The seamless handover function can be called at any point in time, while one can specify a decision-making handover algorithm to be applied. The different decision-making handover algorithms may apply diverse rules and policies, in order to make decisions. These 


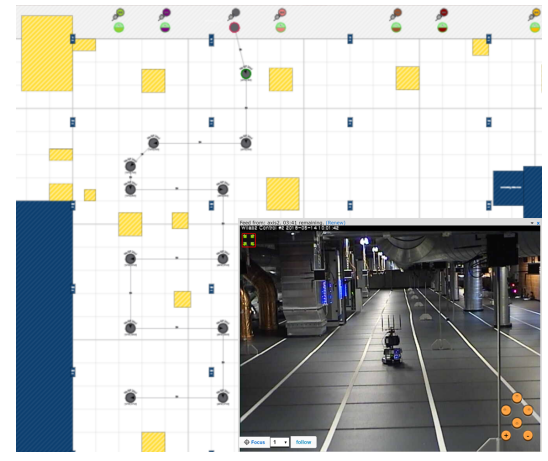

(a) Robot dashboard and camera feed

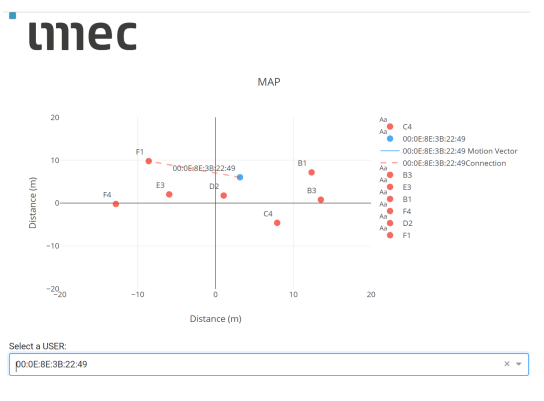

(b) GUI map

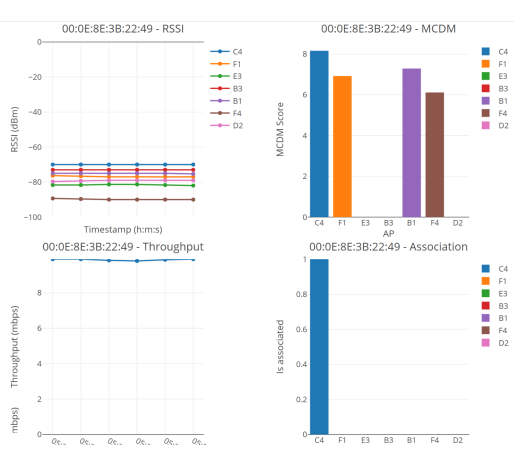

(c) GUI graphs

Figure 1: Mock-up of the GUI and w-iLab.t interface

rules and policies are based on a number of metrics exposed by the framework, which can be the received signal strength indicator (RSSI) value between the mobile node and each AP, the throughput requested by each mobile node, the load of each AP related to the throughputs of associated mobile nodes, the location information of each mobile node and AP, the mobility pattern of each mobile node, and the distance between each node in the network. All this rich information is relevant in the handover decision making process, and can be used by the decision-making handover algorithm to make a smarter handover decision.

For this demo, we have devised 4 decision-making handover algorithms which can be showcased. The first algorithm is the MAX RSSI which monitors the RSSI value of the mobile node to each AP, and hands over the mobile node to the AP with the highest RSSI value at any point in time. PRAHA and ADNA are two similar algorithms, which take into account the location information of the APs and mobile nodes, mobility pattern of the mobile nodes, and the throughput load of the AP when making the handover decision. Both PRAHA and ADNA algorithms take into account to which APs the mobile node is moving towards to, as well as to choose the less loaded APs first. All this information is combined to produce a score for each AP, which represents how good of a candidate a particular AP is for the mobile node to be handed over to. Finally, we have a fourth, artificial intelligence (AI) based algorithm that uses deep neural networks to predict the mobile nodes mobility pattern and takes this into account for the handover decision.

\section{VISUALIZATION}

The whole framework runs in a real-life testbed called wiLab.t, [1]. The w-iLab.t is an experimental, generic, heterogeneous wireless testbed for development and testing of wireless applications via an intuitive web-based interface. It is equipped with fixed wireless nodes and mobile wireless robots, which make it a great environment for testing wireless handovers. The testbed comes equipped with a robot dashboard, shown on figure 1a. The mobility pattern for the robots is defined here. Also, when the experiment starts running, you can view the movement of the robots via a live camera feed from the testbed.

In the demo, we choose a predefined mobility path for the robots or create a new one using the robot dashboard. Using the robot dashboard and live camera feed coming from the testbed, we will be able to monitor where the robots are. On the other side, the framework also has its own graphical user interface (GUI), shown on figures $1 \mathrm{~b}$ and $1 \mathrm{c}$. The first figure shows a map with the locations of the APs (shown as red dots) and mobile nodes (shown as blue dots), that are calculated by the framework itself. On the map itself, one can check to which AP the robot is currently associated to by the means of a red dotted line connecting the robot to the AP. The second figure shows all the different graphs, with different metrics that can be monitored through the framework. Using such a solution, different decision-making handover algorithms can be devised in the framework by using the exposed metrics. They can then be validated in the testbed by monitoring the different metrics and QoS parameters on the GUI. During the demo, we will be able to run a number of experiments with different handover algorithms, show how they work and show how they impact the mobile node's QoS parameters. The decision-making handover algorithms show how information such as the location, mobility patterns and throughput are relevant for a more intelligent handover decision.

\section{REFERENCES}

[1] imec - IDLab w-iLab.t Testbed. 2018. http://doc.ilabt.imec.be/ilabtdocumentation/. (2018). 
A large-scale demonstration of SDN-based handover management

SIGCOMM Posters and Demos '18, August 20-25, 2018, Budapest, Hungary

[2] Institute of Electrical and Electronics Engineers (IEEE). 2012. IEEE Standard for Information technology-Telecommunications and information exchange between systems Local and metropolitan area networks-Specific requirements Part 11: Wireless LAN Medium Access Control (MAC) and Physical Layer (PHY) Specifications. (2012). https://doi.org/10.1109/IEEESTD.2012.6178212

[3] R Riggio. 2016. Demo: The EmPOWER mobile network operating system. 10th ACM International Workshop on Wireless Network Testbeds, Experimental Evaluation, and Characterization, WiNTECH 2016 03-07Octo (2016), 87-88. https://doi.org/10.1145/2980159.2980173

[4] Roberto Riggio, Karina Mabell Gomez, Tinku Rasheed, Julius SchulzZander, Slawomir Kuklinski, and Mahesh K. Marina. 2014. Programming Software-Defined wireless networks. 10th International Conference on Network and Service Management (CNSM) and Workshop (nov 2014), 118-126. https://doi.org/10.1109/CNSM.2014.7014148

[5] Anatolij Zubow, Sven Zehl, and Adam Wolisz. 2016. BIGAP - Seamless handover in high performance enterprise IEEE 802.11 networks. In 2016 IEEE/IFIP Network Operations and Management Symposium. IEEE, 445-453. https://doi.org/10.1109/NOMS.2016.7502842 